\title{
Two Cases of Neonatal Lupus and Literature Review
}

\author{
Dong-Hyun Kim', Seung-Ju Lee', Tae-Ho Kim', Gyeong-Hee Yoo', Ji-Yeon Lee', Sung-Yul Lee², Sung-Hae Chang ${ }^{3}$, Hyun-Ju Lee4, \\ Soon-Auck Hong ${ }^{4}$, Joon-Soo Park'
}

${ }^{1}$ Department of Pediatrics, ${ }^{2}$ Department of Dermatology, ${ }^{3}$ Division of Rheumatology, Department of Internal Medicine, and ${ }^{4}$ Department of Pathology, Soonchunhyang University College of Medicine, Cheonan, Korea

\begin{abstract}
Neonatal lupus is a rare rheumatic disease. Clinical manifestations include characteristic annular or macular rashes, congenital heart block, cytopenias, and hepatitis. Neonatal lupus is caused by transmission of maternal immunoglobulin $\mathrm{G}$ autoantibodies such as anti-SSA/Ro antibody or anti-SSB/La antibody to the fetus through the placenta. We report two cases of neonatal lupus. The first case refers to an 18-day-old male with annular rashes on both cheeks, neutropenia, positive tests for antinuclear antibody, anti-SSA/ Ro antibody, and anti-SSB/La antibody. His mother was diagnosed with systemic lupus erythematosus characterized by positive tests for antinuclear antibody, anti-SSA/Ro antibody, and anti-SSB/La antibody. The second case represents a 32-day-old female with annular rash on both hands, soles, and the genital area, neutropenia, hepatitis, positive tests for antinuclear antibody, and antiSSA/Ro antibody. Skin punch biopsy was conducted. Her mother did not have history of connective tissue diseases. We referred her mother to the division of rheumatology of the department of internal medicine. The mother was suspected with primary Sjögren's syndrome because of arthralgia and dry eye symptoms with positive tests for antinuclear antibody, anti-SSA/Ro antibody, anti-SSB/ La antibody, and rheumatoid factor. It is necessary to suspect neonatal lupus in neonates or infants with characteristic annular rash with or without maternal history of connective tissue disorders.
\end{abstract}

Keywords: Neonatal lupus; Autoantibodies; Exanthema

\section{INTRODUCTION}

Neonatal lupus (NL) is a rare disease with a prevalence of 1 out of 20,000 live births. The disease occurs when autoantibodies such as anti-SSA/Ro antibodies (Ab) or anti-SSB/La Ab are transmitted from the mother to the fetus through the placenta [1-3]. NL appears in babies born to mothers with systemic lupus erythematosus (SLE), Sjögren's syndrome, and other collagen vascular diseases [2].

Symptoms of NL include annular skin rash, anemia, neutropenia, hepatitis, and congenital heart block. Diagnosis is based on clinical symptoms and the blood tests including autoantibodies of mother and baby. Treatment depends on the symptoms, and cardiac function should be monitored periodically to confirm cardiac complications. NL usually has a favorable prognosis, and in most cases, it improves naturally. However, a congenital heart block often requires a pacemaker. About $60 \%$ of patients with congenital heart block require a pacemaker and deaths may result following the failure of the pacemaker [1].

We experienced two cases of NL and report it with a review of literature (Table 1).

\section{CASE REPORT}

\section{Case 1}

A 23-day-old male neonate visited the pediatric outpatient clinic in Soonchunhyang University Cheonan Hospital because of a red, round ring-shaped rash on both cheeks. There was no specific family history. This pediatric patient was born healthy without any problems with a weight of $2,860 \mathrm{~g}$ on the third day of the 40 th
Correspondence to: Joon-Soo Park

Department of Pediatrics, Soonchunhyang University Cheonan Hospital, 31 Suncheonhyang 6-gil, Dongnam-gu, Cheonan 31151, Korea

Tel: +82-41-570-2163, Fax: +82-41-572-4996, E-mail: pjstable@schmc.ac.kr

Received: Apr. 16, 2018 / Accepted after revision: Jun. 8, 2018
(C) 2018 Soonchunhyang Medical Research Institute This is an Open Access article distributed under the terms of the Creative Commons Attribution Non-Commercial License (http://creativecommons.org/licenses/by-nc/4.0/). 
Table 1. Two cases of neonatal lupus: a clinical profile

\begin{tabular}{lll}
\hline Variable & \multicolumn{1}{c}{ Case 1} & \multicolumn{1}{c}{ Case 2} \\
\hline Sex & Male & Female \\
Age & 25 days old & 32 days old \\
Skin rash & Annular rash on both cheeks & Annular rash on both hands, sole and genital area \\
Hematologic problem & Neutropenia & Neutropenia, anemia \\
Aspartate aminotransferase/alanine aminotransferase (IU/L) & Not elevated & Not elevated \\
Congenital heart block & None & None \\
Antinuclear antibody & Positive & Positive \\
Patient's autoantibodies & Anti-SSA/Ro (+), anti-SSB/La (+) & Anti-SSA/Ro (+), anti-SSB/La (-) \\
Maternal autoantibodies & Anti-SSA/Ro (+), anti-SSB/La (+) & Anti-SSA/Ro (+), anti-SSB/La (+) \\
Maternal rheumatic disease & Systemic lupus erythematosus & Sjögren's syndrome
\end{tabular}
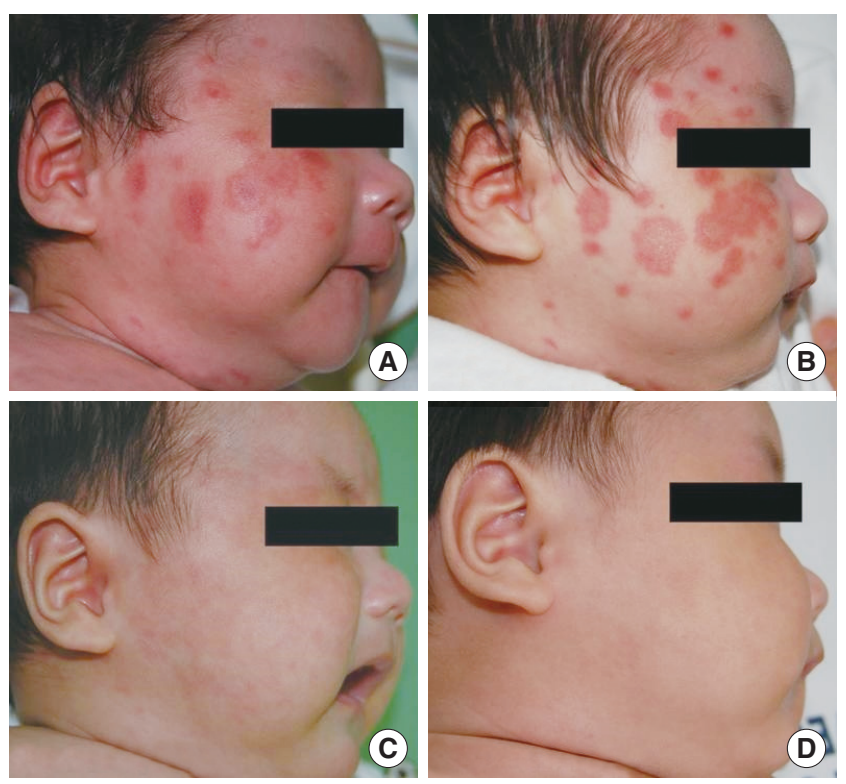

Fig. 1. A 4-week-old male baby showed annular rash on both cheeks on the 18th day after birth. His mother had systemic lupus erythematosus history. The skin lesion improved on the 88th day after birth. (A) 25th day after birth. (B) 28th day after birth. (C) 68th day after birth. (D) 122nd day after birth.

week, but several red papuloid rashes appeared on both cheeks on the 18th day after birth. On the 25th day after birth, the diameter of the rashes ranged from approximately 5 to $15 \mathrm{~mm}$, the central part of the round rash turned purple. The outer rim of the rash slightly bulged, changed to the shape of a target or round ring, and some rashes fused together (Fig. 1).

The absolute neutrophil count (ANC) was decreased to 710/ $\mathrm{mm}^{3}$ in complete blood count. The blood chemistry test showed that aspartate aminotransferase (AST)/alanine aminotransferase (ALT) was within the normal range of 46/25 IU/L (normal range, 22-63/12-45 IU/L), and antinuclear antibody (ANA) was positive (qualitative test, speckled type). At that time, the patient was diagnosed with erythema multiforme and was administered antihistamine. The color of the rash became faint and slightly improved.

On the 38th day after birth, the color of rashes on both cheeks became prominent and darker, and the rashes worsened again. In the blood test conducted at that time, anti-SSA/Ro Ab was 191.96 $\mathrm{U} / \mathrm{mL}$ ( $<7$, negative; 7-10, equivocal; $>10$, positive) and anti-SSB/ $\mathrm{La} \mathrm{Ab}$ was $167.26 \mathrm{U} / \mathrm{mL}$ ( $<7$, negative; $7-10$, equivocal; $>10$, positive), and the results were positive. We also found that the mother of the boy had been diagnosed with SLE at the other hospital's division of rheumatology, and the mother's blood test showed antiSSA/Ro Ab positive (274.50 U/mL [ $<7$, negative; $7-10$, equivocal; $>10$, positive]), and anti-SSB/La Ab positive $(179.06 \mathrm{U} / \mathrm{mL}[<7$, negative; 7-10, equivocal; $>10$, positive]). Based on these findings, we diagnosed the boy as NL. Echocardiography and electrocardiography (ECG) findings were normal.

Skin rash disappeared without scarring on the 122nd day after birth, and ANA was normal at age 3 months, and neutropenia was normal at age 6 months. Anti-SSA/Ro Ab and anti-SSB/La Ab were positive at age 6 months and changed to negative with $5.4 \mathrm{U} /$ $\mathrm{mL}$ and $5.2 \mathrm{U} / \mathrm{mL}$ at age 12 months, respectively. The boy is currently undergoing follow-up observations.

\section{Case 2}

A 56-day-old female infant visited the pediatric outpatient clinic because of a round-shaped rash around the hands, soles and the genital area. The mother of the girl had no history of connective tissue disease. The patient was born healthy at second day of 38th week, and weighed 3,380 g. The skin rash started around the hands, soles and the genital area on the 32nd day after birth. She was admitted to our neonatal intensive care unit with broncho- 
pneumonia in addition to skin rash on the 33rd day after birth.

At the time of admission, anemia (hemoglobin, $9.5 \mathrm{~g} / \mathrm{dL}$ [normal lower limit, $10.0 \mathrm{~g} / \mathrm{dL}$; hematocrit, 27.1\% [normal lower limit, 31\%]; neutropenia [ANC, 780/ $\mathrm{mm}^{3}$ ]; and increased ALT [AST/ ALT, 61/54 IU/L; normal value, 22-63/12-45 IU/L]) were detected. Respiratory syncytial virus was detected via polymerase chain reaction. The girl was discharged after improvement in the respiratory symptoms whereas the skin rash persisted.

On the 56th day after birth, round-shaped skin rashes appeared on the forehead, neck, palms, and soles (Fig. 2). At this time, he-
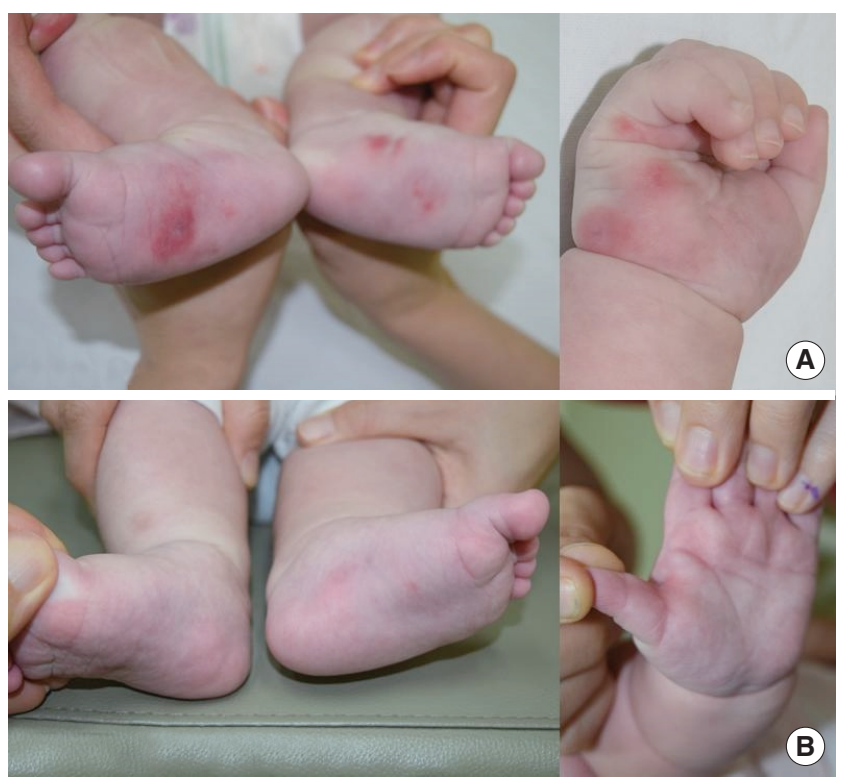

Fig. 2. A 1-month-old female baby with annular rash on the palm, sole and genital area on the 32nd day after birth. She was diagnosed with neonatal lupus on the 66th day after birth. The rash disappeared on the 120th day after birth. After this baby's diagnosis, the baby's mother also visited division of rheumatology and was diagnosed with Sjögren's syndrome. (A) 97th day after birth. (B) 150th day after birth. moglobin was $9.5 \mathrm{~g} / \mathrm{dL}$ (normal lower limit, $10.0 \mathrm{~g} / \mathrm{dL}$ ) and hematocrit was 27.6\% (normal lower limit, 31\%), and ANC was reduced to $900 / \mathrm{mm}^{3}$, AST/ALT increased to $68 / 71 \mathrm{IU} / \mathrm{L}$, and ANA was positive (qualitative test, speckled type).

The blood test results on the 66th day after birth showed positive for anti-SSA/Ro $\mathrm{Ab}$ (>600 U/mL), and negative for anti-SSB/ $\mathrm{La} \mathrm{Ab}$ and U1-ribonucleoprotein $\mathrm{Ab}$. We suspected NL and transferred the baby's mother to the division of rheumatology for evaluation of connective tissue disease. She had not visited a rheumatologist until then.

We performed a skin punch biopsy on the patient on the 75th day after birth. It revealed the hyperkeratosis, basal cell layer hydropic degeneration, and increase in dermal mucin, consistent with NL findings (Fig. 3). The mother presented dry eye symptoms and arthralgia, with results indicating positive for ANA (1:80), rheumatoid factor $(228.8 \mathrm{IU} / \mathrm{mL}$; reference range, $0-15 \mathrm{IU} /$ $\mathrm{mL})$, anti-SSA/Ro $\mathrm{Ab}(>600 \mathrm{U} / \mathrm{mL}$; reference range, $<7 \mathrm{U} / \mathrm{mL})$, and anti-SSB/La $\mathrm{Ab}(26 \mathrm{U} / \mathrm{mL}$; reference range, $<7 \mathrm{U} / \mathrm{mL})$, respectively. Therefore, she was diagnosed as the primary Sjögren's syndrome and prescribed anti-malarial drugs.

The skin symptoms were disappeared on the 120th day after birth. AST/ALT, ANC, and ANA levels were normalized at 7 months of age. The patient tested positive for anti-SSA/Ro Ab up to 10 months of age and turned negative $(<1 \mathrm{U} / \mathrm{mL})$ at age 18 months. ECG conducted till the age of 6 months revealed no congenital heart block. The patient is currently under follow-up in the outpatient clinic.

\section{DISCUSSION}

NL is a rare disease in infants caused by autoantibodies trans-
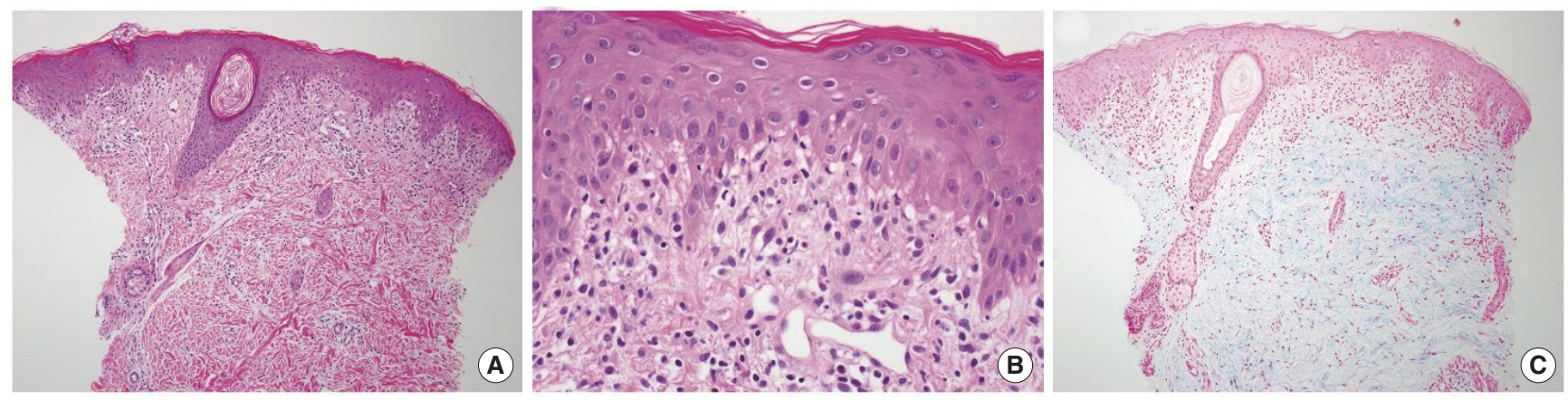

Fig. 3. Histologic features from case 2. (A) Perivascular lymphocytic infiltration with hyperkeratosis and keratin plugging was observed (H\&E, $\times 40)$. (B) High power view showed liquefactive degeneration of the basal layer with a few cytoid bodies was noted $(\mathrm{H} \& \mathrm{E}, \times 200)$. (C) Alcian blue staining demonstrated increased dermal $\operatorname{mucin}(\times 40)$. 
mitted from the mother. Ninety-eight percent of the causative $\mathrm{Ab}$ include anti-SSA/Ro Ab and anti-SSB/La Ab, in addition to U1-ribonucleoprotein $\mathrm{Ab}$ [1-3]. These immunoglobulin $\mathrm{G} A \mathrm{~b}$ migrate to the fetus via placenta, causing fetal tissue damage and NL symptoms.

NL is an entirely different disease from SLE [1]. NL is common in females rather than males, and typical symptoms include annular or spotty rashes on the face (especially periorbital), torso, and scalp [2]. Symptoms other than skin include the hepatitis, neurological abnormality, defective spleen, congenital heart block, and hematocytopenia such as anemia, neutropenia, and thrombocytopenia [1-4]. In approximately $10 \%$ of the cases, skin symptoms and cardiac problems occur concomitantly [2].

The rash is detected in $15 \%-25 \%$ of children with NL [4]. It appears during the first 3 months after birth, lasts for a mean of 4 months, and disappears spontaneously by 6-8 months of age as the maternal Ab in baby's circulation disappear. However 10\%-25\% of patients manifest residual skin abnormlities such as telangiectasias, dyspigmentation, pitting, scarring, and skin atrophy [5].

Congenital heart block ranges from the prolonged PR interval to the complete heart block. During development of the heart, Ro and La antigens can be exposed to the surface of the cardiac cells near the atrioventricular node, and these antigens react with the autoantibodies received from the mother. This antigen-Ab binding causes a localized immune response, that induces fibrosis in the conduction system and challenges such as heart block [6,7]. It is usually confirmed by fetal echocardiography at 18 to 24 weeks of gestation. If the mother is anti-SSA/Ro Ab positive, it is found in $2 \%$ of fetuses. In case of anti-SSA/Ro $\mathrm{Ab}$ and anti-SSB/La Ab positive, it is found in $5 \%$ of fetuses $[5,8]$. While the non-cardiac symptoms are usually reversible, the congenital heart block is permanent and approximately $20 \%$ may be accompanied by cardiomyopathy and death from heart failure [2].

Postnatal diagnosis of NL is based mainly on clinical symptoms and blood tests including autoantibodies such as anti-SSA/Ro Ab, anti-SSB/La Ab, or anti-U1- ribonucleoprotein Ab of mother and baby. Evaluation of the anemia, thrombocytopenia, hepatitis, and hyperbilirubinemia is also indicated [9].

NL should be distinguished from diseases that may show coinshaped rash in newborns such as drug side effects, urticaria, pemphigoid, and tinea. The pathologic findings of NL are similar to subacute lupus erythematous. Its findings include hyperkeratosis, liquefactive degeneration, follicular plugging, and dermal perivas- cular infiltrates with lymphocyte predominance, although skin biopsy is not always needed $[10,11]$. If maternal anti-SSA/Ro Ab or anti-SSB/La Ab are positive, fetal echocardiograms should be routinely monitored on a weekly or bi-weekly basis starting from 1618 weeks of gestation [12].

Fluorinated corticosteroids such as dexamethasone and betamethasone may be used in prenatal mothers to prevent congenital heart block in fetal period. However, risks such as maternal infection, hypertension, avascular osteonecrosis, insulin resistance, gestational diabetes, oligoamnios, and fetal growth retardation are increased. In addition, intravenous immunoglobulin injections, exchange transfusions, and hydroxychloroquine may be used $[13,14]$. After birth, echocardiography and electrocardiogram should be conducted at 1 month and 1 year after birth [15] despite normal fetal echocardiogram. In case of complete heart block, a pacemaker insertion may be warranted [1].

Conservative treatments are indicated depending on the symptoms. Most skin symptoms improve without scarring, without the need for special treatment. Topical steroid ointment, anti-malarials, and laser treatments are available. In addition, sunscreens and protective clothing are beneficial because exposure to sunlight can worsen the lesion [1].

In the cases reported here, the authors administered systemic steroids considering the possibility of heart block or invasion of internal organs. In the future, guidelines for the use of steroids are needed. It is difficult to find literature about when anti-SSA/Ro Ab and anti-SSB/La Ab turn negative. In case 1, anti-SSA/Ro Ab and anti-SSB/La Ab were negative at age 12 months. In case 2, anti$\mathrm{SSA} / \mathrm{Ro} \mathrm{Ab}$ was positive until age 10 months and turned negative at age 18 months.

Medical history of the mother is crucial for the diagnosis, but caution is needed because approximately half of the mothers have no symptoms at the time of their diagnosis [2]. In case 1, we didn't know mother's SLE history at first, but we came to know that through specific question. In case 2 , NL diagnosis of the baby was followed by referral of the mother to the division of rheumatology. The mother was diagnosed with primary Sjögren's syndrome, because of arthralgia and dry eye symptoms with positive ANA, anti-SSA/Ro Ab, anti-SSB/La Ab, and rheumatoid factor.

We should suspect NL when neonates or infants manifest typical skin rash, with or without a maternal history of connective tissue disorders. 


\section{REFERENCES}

1. Hon KL, Leung AK. Neonatal lupus erythematosus. Autoimmune Dis 2012;2012:301274.

2. Perez MF, Torres ME, Bujan MM, Lanoel A, Cervini AB, Pierini AM. Neonatal lupus erythematosus: a report of four cases. An Bras Dermatol 2011;86:347-51.

3. Ramphul K, Mejias SG, Ramphul-Sicharam Y. Cutaneous neonatal lupus erythematosus: a case report. Cureus 2018;10:e2212.

4. Silverman E, Jaeggi E. Non-cardiac manifestations of neonatal lupus erythematosus. Scand J Immunol 2010;72:223-5.

5. Nasef N, Hafez M, Bark A. Neonatal lupus erythematosus. J Neonatol Clin Pediatr 2014;1:002.

6. Capone C, Buyon JP, Friedman DM, Frishman WH. Cardiac manifestations of neonatal lupus: a review of autoantibody-associated congenital heart block and its impact in an adult population. Cardiol Rev 2012;20: 72-6.

7. Izmirly PM, Buyon JP, Saxena A. Neonatal lupus: advances in understanding pathogenesis and identifying treatments of cardiac disease. Curr Opin Rheumatol 2012;24:466-72.

8. Yildirim A, Tunaoolu FS, Karaaoac AT. Neonatal congenital heart block. Indian Pediatr 2013;50:483-8.

9. Kim KR, Yoon TY. A case of neonatal lupus erythematosus showing tran- sient anemia and hepatitis. Ann Dermatol 2009;21:315-8.

10. Penate Y, Guillermo N, Rodriguez J, Hernandez-Machin B, Montenegro T, Afonso JL, et al. Histopathologic characteristics of neonatal cutaneous lupus erythematosus: description of five cases and literature review. J Cutan Pathol 2009;36:660-7.

11. Barcelos A, Fernandes B. Cutaneous manifestations of neonatal lupus: a case report. Acta Reumatol Port 2012;37:352-4.

12. Donofrio MT, Moon-Grady AJ, Hornberger LK, Copel JA, Sklansky MS, Abuhamad A, et al. Diagnosis and treatment of fetal cardiac disease: a scientific statement from the American Heart Association. Circulation 2014;129:2183-242.

13. Breur JM, Visser GH, Kruize AA, Stoutenbeek P, Meijboom EJ. Treatment of fetal heart block with maternal steroid therapy: case report and review of the literature. Ultrasound Obstet Gynecol 2004;24:467-72.

14. Kelly EN, Sananes R, Chiu-Man C, Silverman ED, Jaeggi E. Prenatal antiRo antibody exposure, congenital complete atrioventricular heart block, and high-dose steroid therapy: impact on neurocognitive outcome in school-age children. Arthritis Rheumatol 2014;66:2290-6.

15. Friedman D, Sadun RE, Ardoin SP, Schanberg LE. Neonatal lupus. In: Kliegman RM, Stanton BF, St. Geme JW, Schor NF, Behrman RE, editors. Nelson textbook of pediatrics. 20th ed. Phialdelphia (PA): Elsevier; 2016. p. 1180-1. 